\title{
UNA RESPONSABILIDAD ESCOLAR OLVIDADA: EL DESARROLLO DEL COMPONENTE LÉXICO
}

\author{
Marta Rojas Porras \\ Docente de la Universidad de Costa Rica.
}

"Soy hombre, es decir, animal con palabras, $Y$ exijo, por lo tanto, que me dejen usarlas"

Jorge Debravo, Hombre

\begin{abstract}
Resumen: El artículo plantea la necesidad de atender, de manera planificada, el desarrollo del léxico en el sistema educativo costarricense. Analiza resultados derivados de una muestra nacional de 1712 textos escritos por escolares de cuarto y sexto años. Expone datos relativos al promedio de lemas por redacción, densidad léxica y organiza, en campos conceptuales, las palabras que obtuvieron frecuencias iguales o superiores a cien (100F). Además, propone parámetros para medir densidad léxica en el primero y segundo ciclos de la Educación General Básica. Concluye que es muy probable que la pobreza léxica encontrada en el estudiantado afecte negativamente la capacidad potencial para referirse al mundo, para mostrar sus relaciones y para captar abstracciones y que limite los aprendizajes de las diversas asignaturas del currículo escolar.
\end{abstract}

Palabras clave: Léxico básico, lingüística aplicada, aprendizaje, lengua materna.
El epígrafe reza una plegaria que los niños y las niñas costarricenses podrían estar clamando a gritos y una responsabilidad que los profesionales de la lingüística aplicada y los educadores debemos asumir con prioridad.

Es ya un lugar común referirse a las limitaciones que respecto del uso del lenguaje se manifiestan en los escolares y la preocupación que esta situación plantea, pues aparte del interés que éste reviste en tanto factor que ayuda a la integración del individuo a la sociedad, se relaciona estrechamente con el pensamiento. Además, el léxico, componente de la lengua que se aborda en este artículo, se vincula directamente con el desarrollo conceptual: en él se presenta con mayor claridad la característica de intelectuación, conectada con las posibilidades de reflexión, aprendizaje y pensamiento. (Rojas y Umaña:1986).

Según López Morales (1984), para generar nuevas hipótesis lingüísticas en el niño, la escuela tiene que proporcionar los medios para que el alumno reformule sus hipótesis provisionales. En este 


\begin{abstract}
This article states the need to help, in quite a well planned fashion, lexical development in the Costa Rican educational system. It analyzes the results derived from a national sample of 1712 texts written by four-and six-graders. It shows data on the average of lemmas per composition and on lexical density. It also analyzes into conceptual fields those words equal or over 100 in frequency 100F). It proposes some parameters to measure lexical density in Cycles I and II of the Basic General Education. It concludes that it is very likely that the lexical poverty found in the students affects negatively their potential ability to refer to the world, to show relationships and to grasp abstractions. Such poverty would limit the learning of different subjects in the primary school curriculum.
\end{abstract}

Key words: Basic lexicon, applied linguistics, learning, mother tongue. sentido es necesario saber cuáles hipótesis están formadas, cuáles deben ser reforzadas, cuáles necesitan reformulación y cuáles deben ser formadas.

Para identificar estas hipótesis, en cuanto al componente léxico de la lengua, los estudios de léxicos básicos y de léxicos disponibles resultan un instrumental fundamental, pues dan cuenta del vocabulario que los hablantes usan (básicos) y de los contextos comunicativos en los que lo actualizan (disponibles). Siendo así, estos estudios resultan un instrumental teórico para tratar de mejorar la práctica educativa a partir de referentes reales y no de decisiones antojadizas.

Estos recuentos constituyen un aporte para la labor del sistema educativo en su responsabilidad de preparar inventarios léxicos, materiales didácticos, libros de texto y prácticas que enfrenten al estudiantado a experiencias lingüísticas que actúen como estímulos apropiados para su desarrollo.

Como queda dicho, para una intervención posterior se hace necesario reconocer el léxico que se utiliza. Al respecto, en Costa Rica vale la pena destacar la labor pionera y conjunta del doctor Víctor Manuel Sánchez y de la doctora Marielos Murillo, en el programa ELEHXICÖS del Instituto de Investigaciones Lingüísticas de la Universidad de Costa Rica, quienes han definido léxicos básicos y disponibles para la educación preescolar y para la escuela primaria. Además, con la colaboración del Dr. Sánchez, la proponente de este escrito, desde el Instituto de Investigación en Educación, también de la Universidad de Costa Rica, ha trabajado la investigación Léxico básico escolar costarricense: diccionario de frecuencias (Rojas: 2002), de la que se derivan los datos que se presentarán en este artículo.

En el estudio mencionado se recogieron 1712 redacciones de estudiantes de cuarto y sexto años (considerando al cuarto como nivel de salida del primer ciclo y al sexto, como el de segundo ciclo), en un muestreo al azar que abarcó las 
19 regiones educativas del país. De cada región se seleccionaron dos escuelas: una urbana y otra rural, de manera que la muestra quedó conformada por 38 escuelas. En cada escuela se seleccionó un grupo de cuarto y otro de sexto. En cada escuela se realizaron dos sesiones: una para cada grupo. Se le entregó a cada niño o niña una hoja en la que debía anotar el nombre de la escuela, año cursado y sexo. Se les pidió que escribieran una redacción de tema libre y que le pusieran un título. En general, se trató de que el clima de la clase fuera propicio para que escribieran con libertad. Sólo si se consideraba que su actitud interfería con el trabajo de los niños o las niñas, se le pedía al maestro o a la maestra que se ausentara del grupo, lo cual ocurrió en muy pocas ocasiones.

Las 1712 se transcribieron de manera textual en un procesador de textos y, posteriormente el léxico que utilizaban se organizó en listas lematizadas. Éstas consideran la unidad léxica y las formas flexivas que representan como una sola unidad. (Por ejemplo, 'he comido', 'comió', 'comieron' se cuentan en el lema 'comer'.). Además, y como es usual en este tipo de estudio, se recuentan sólo las palabras "contenido" (sustantivos, verbos, adjetivos y adverbios) y no las funcionales (conjunciones, preposiciones, contracciones, interjeccciones). (Lyons:1983). Posteriomente los datos fueron procesados para recoger las estadísticas.

Los momentos anotados anteriormente representan procesos muy exhaustivos que requirieron de gran cantidad de tiempo y de personal de apoyo.

El objetivo de la publicación de este artículo es mostrar algunos de los datos de esta investigación y reflexionar al respecto. Además, ofrecer un primer acercamiento a la definición de parámetros de densidad léxica para el primer y segundo ciclo de la educación básica costarricense.

Los resultados se mostrarán en cuadros que presentan el promedio de ocurrencias y lemas por redacción, según niveles (cuarto y sexto); según zona (rural y urbana) y según sexo (masculino y femenino). Se organizará la propuesta de parámetros de densidad y se anotarán las palabras que obtuvieron una frecuencia igual o superior a 100 F. Estas se organizarán en campos conceptuales.

\section{Referentes conceptuales}

\section{El léxico}

El léxico es un sistema de unidades mínimas portadoras de significado y relacionadas entre sí por reglas combinatorias, las que permiten y controlan la creación de nuevas palabras. Es decir, es un todo unitario definido por reglas semánticas, sintácticas y pragmáticas.

El léxico es el componente de la lengua referido al vocabulario y a sus posibilidades de variedad (cantidad del repertorio y uso de sinónimos), propiedad (adecuación al contexto lingüístico y pragmático) y exactitud (selección de los significados que mejor expresen lo que se quiere expresar).

Como componente de la lengua, el léxico es sujeto de la variación lingüística, tanto en términos geográficos como sociales y estilísticos. Por esto se pueden definir repertorios léxicos de Guanacaste, de América, de los escolares, de los adolescentes, de los campesinos, del fútbol, de los textos didácticos, del informe científico, etc.

El hablante maneja dos tipos de vocabulario: uno pasivo, más amplio y relacionado con la comprensión; y otro activo, más limitado, asociado a la producción.

La lengua académica (la estándar) se caracteriza por un vocabulario preciso, de terminología creciente, de expresiones claramente diferenciadas y de vocablos abstractos y genéricos (Garvin y Mathiot:1974). A lograr estas condiciones debe tender la enseñanza y aprendizaje del vocabulario, pues el uso de repertorios restringidos a muy pocos significados constituye un obstáculo en los procesos educativos, en la 
promoción social y en el acceso a órdenes superiores del pensamiento.

\section{Léxicos básicos y disponibles}

Los léxicos básicos ofrecen perfiles muy adecuados del vocabulario usual de una comunidad lingüística dada, por lo que, sin duda, constituyen un valiosísimo instrumento para la planificación léxica en la escuela. Muestran una proporción real del uso de las diferentes clases de palabras.

Por su parte, la disponibilidad léxica señala el vocabulario concreto que el hablante utiliza en contextos comunicativos determinados.

Los léxicos básicos recogen las palabras estables (las que tienen mayor posibilidad de aparecer en circunstancias diferentes), mientras que los disponibles muestran los más inestables (sus posibilidades se refieren a contextos específicos). Estos son, entonces, criterios complementarios para constituir el léxico fundamental.

\section{Los recuentos de vocabulario}

Los recuentos de frecuencias son cálculos inferenciales del vocabulario, basados en la relación vocablo/palabra (en nuestros términos: ocurrencia de palabra/lema), es decir, en la cantidad de veces que aparece una misma palabra en proporción al número total de palabras emitidas por una muestra de sujetos. Se trata, por tanto, de recuentos de vocabulario activo. A las listas de palabras que contienen los vocabularios de frecuencias se les denomina vocabularios básicos o usuales. (Fernando Justicia:1995).

\section{Densidad léxica}

Densidad léxica es la medida que se basa en determinar cuántas palabras aparecen en un segmento determinado de un texto. Para controlar la variable "extensión del texto", se ha determinado que éste conste de 100 palabras ocurrencia (Ávila:1991).
La fórmula que se utiliza es:

$\mathrm{DL}=\mathrm{L} / \mathrm{O}$ (en textos de 100 palabras contenido)

Donde:

$\mathrm{D}=$ densidad léxica

$\mathrm{L}=$ lema (palabra tipo: las que registra el diccionario, por ej. 'comer')

$\mathrm{O}=$ ocurrencia de palabra (palabra con sus respectivas flexiones, por ej., 'comiendo')

A mayor densidad léxica de un texto, el emisor presenta más riqueza léxica y viceversa. (Sánchez:1998).

\section{Resultados}

\section{Promedios de lemas por redacción}

En la investigación que referimos se obtuvo, como ya se dijo, un total de 1712 redacciones y un recuento de lemas o palabras tipo constituido por 5234 lemas.

Los siguientes cuadros resumen los resultados generales respecto al promedio de lemas por redacción.

Cuadro 1

Promedio de lemas por redacción, comparación general por grado, zona y sexo

\begin{tabular}{llllll}
\hline Zona & & Grado & & Sexo & \\
\hline Rural & 57 & Cuarto & 45 & Femenino & 68 \\
Urbana & 69 & Sexto & 77 & Masculino & 64 \\
\hline
\end{tabular}

En general la producción de lemas por sexo es relativamente parecida, se diferencia más entre urbano-rural y se acrecienta considerablemente en sexto. Esta última variable parece mostrar el efecto de la escuela, otros factores asociados a la experiencia y al crecimiento en cuanto al desarrollo léxico. 
Cuadro 2

Promedio de lemas por redacción, comparación por grado, en cuanto a zona y sexo

\begin{tabular}{lllll}
\hline Grado & Zona & \multicolumn{3}{l}{ Sexo } \\
\hline \multirow{2}{*}{ Cuarto } & Rural & 36 & Femenino & 48 \\
& Urbana & 51 & Masculino & 43 \\
\hline \multirow{2}{*}{ Sexto } & Rural & 74 & Femenino & 82 \\
& Urbana & 78 & Masculino & 73 \\
\hline
\end{tabular}

En cuanto a rural-urbana, la diferencia es más pronunciada en los niveles inferiores y se va equilibrando en sexto. Lo contrario ocurre con la variable sexo que se va diferenciando conforme se avanza en escolaridad. El primer dato podría relacionarse con el efecto estandarizador del sistema escolar; el segundo con una estimulación diferenciada.

\section{Cuadro 3}

Promedio de lemas por redacción comparación de la variable sexo, según zona

\begin{tabular}{lll}
\hline Zona & Sexo & \\
\hline \multirow{2}{*}{ Rural } & Femenino & 58 \\
& Masculino & 57 \\
\hline \multirow{2}{*}{ Urbano } & Femenino & 71 \\
& Masculino & 68 \\
\hline
\end{tabular}

Respecto de la variable sexo, la diferencia en cantidad de vocabulario se marca más en lo urbano a favor de las niñas.
Cuadro 4

Promedio de lemas por redacción comparación por grado, según sexo y zona

\begin{tabular}{llll}
\hline Grado & Zona & Sexo & \\
\hline \multirow{3}{*}{ Cuarto } & Rural & Femenino & 39 \\
& & Masculino & 34 \\
& Urbana & Femenino & 52 \\
& & Masculino & 50 \\
\hline \multirow{3}{*}{ Sexto } & \multirow{2}{*}{ Rural } & Femenino & 81 \\
& & Masculino & 71 \\
& Urbana & Femenino & 82 \\
& & Masculino & 74 \\
\hline
\end{tabular}

En cuarto año, la diferencia en rural-urbano es muy marcada a favor de lo urbano, mientras que las diferencias en variedad léxica entre hombres y mujeres son muy leves.

En sexto, lo rural y lo urbano tienden a compensarse; pero las diferencias en la cantidad de palabras utilizadas entre niños y niñas tienden a marcarse.

\section{Una aproximación tentativa a la definición de parámetros de densidad léxica para el primer y segundo ciclos}

En la investigación no se controló la variable "ocurrencias de palabras por redacción”, pues en principio no se pensó en medir densidad léxica. No obstante, a pesar de que este hecho plantea una limitación importante, pues a mayor cantidad de texto, mayores son las probabilidades de repetición 
de palabras, se hará un cálculo aproximado y tentativo, el cual debe interpretarse y valorarse con las reservas del caso.

Considérense así los datos del siguiente cuadro.

\section{Cuadro 5}

Promedio de ocurrencias de vocablos y lemas, y densidad léxica aproximada

\begin{tabular}{lccc}
\hline Variable & $\begin{array}{c}\text { Oc. de } \\
\text { vocablo }\end{array}$ & Lema & $\begin{array}{c}\text { Densidad } \\
\text { léxica }\end{array}$ \\
\hline Rural & 110 & 57 & 51,81 \\
Urbana & 143 & 69 & 48,25 \\
Cuarto & 85 & 45 & 52,94 \\
Sexto & 77 & 159 & 48,42 \\
Femenino & 68 & 141 & 48,22 \\
Masculino & 64 & 127 & 50,39 \\
\hline
\end{tabular}

Reiterando la salvedad de que en estos datos no se consideró la "extensión del texto", en 100 ocurrencias de palabras, se observa que aunque escribieron más los de la urbe, lo hacen con mayor variedad léxica los grupos de zonas rurales; los sextos escriben más, pero también repiten más, y los varones escriben menos, no obstante alcanzan mayor densidad léxica.

A pesar de que en todos los grupos se evidencian diferencias en cuanto a la densidad léxica, éstas son muy leves.

De 181 redacciones, correspondientes a un submuestreo del 10,57\% del total, se escogieron 15 redacciones $8,29 \%$, cuyos promedios de ocurrencia de palabras osciló en el rango de 93 a 106. Todas corresponden a sexto año. No fue posible hacerlo con el primer ciclo, pues la mayoría escribió textos muy pequeños. El propósito de este submuestreo fue poder obtener datos más susceptibles de comparación con los parámetros establecidos de 100 y que la variable "extensión del texto" estuviera relativamente controlada. A continuación los datos:
Cuadro 6

Densidad léxica de un submuestreo

\begin{tabular}{rrrr}
\hline & $\begin{array}{c}\text { Ocurrencias } \\
\text { de vocablo }\end{array}$ & Lemas & Densidad \\
\hline 1. & 102 & 59 & 57,84 \\
2. & 99 & 48 & 48,48 \\
3. & 106 & 58 & 54,72 \\
4. & 99 & 53 & 53,53 \\
5. & 93 & 50 & 53,76 \\
6. & 102 & 55 & 53,92 \\
7. & 106 & 64 & 60,37 \\
8. & 103 & 60 & 58,25 \\
9. & 100 & 53 & 53,00 \\
10. & 100 & 43 & 43,00 \\
11. & 96 & 51 & 53,13 \\
12. & 106 & 57 & 53,77 \\
13. & 94 & 61 & 64,89 \\
14. & 95 & 54 & 56,84 \\
15. & 95 & 55 & 57,79 \\
\hline
\end{tabular}

La densidad léxica oscila de 43,00 (la menor) a 64,89 (la mayor).

Sánchez (1998) propone párametros para la densidad léxica en secundaria. Con base en estos parámetros establecidos, tomando en cuenta los datos precedentes y el hecho de que el cuarto año se consideró el nivel de salida del primer ciclo y el sexto el del segundo, nos atrevemos a una propuesta:

\section{Cuadro 7}

Propuesta de parámetros para densidad léxica

\begin{tabular}{llll}
\hline & Tercer ciclo & Segundo ciclo & Primer ciclo \\
\hline $\begin{array}{l}\text { Densidad } \\
\text { rica }\end{array}$ & 66,5 a 70,5 & 60,5 a 66,5 & 55,5 a 60,5 \\
$\begin{array}{l}\text { Densidad } \\
\text { media }\end{array}$ & 60,5 a 64,5 & 55,5 a 60,4 & 50,5 a 55,4 \\
$\begin{array}{l}\text { Densidad } \\
\text { inferior }\end{array}$ & 54,5 a 58,5 & 50,5 a 55,4 & 45,5 a 50,4 \\
\hline
\end{tabular}

Nota: La definición de parámetros para tercer ciclo es la planteada por Sánchez (1998). 
Si con estos parámetros se analizan los datos del submuestreo del cuadro 6 , la densidad léxica obtenida sería:

\section{Cuadro 8}

Calificación, según densidad léxica, de las redacciones del submuestreo

\begin{tabular}{lll}
\hline 2 & Superior & $13,33 \%$ \\
4 & Media & $26,66 \%$ \\
9 & Inferior & $60,00 \%$ \\
\hline
\end{tabular}

Obsérvese cómo la densidad léxica muestra que más de la mitad de los textos son pobres en vocabulario, de lo cual se desprende que es muy probable que manifiesten otras carencias en relación con lo conceptual.

De este submuestreo, el promedio de ocurrencias de palabras fue de 99,73, correspondiente a un promedio de 54,73 lemas, lo cual da una densidad léxica de 54,87 , ubicada en densidad inferior.

Con las limitaciones del caso, analicemos la información del siguiente cuadro:

\section{Cuadro 9}

Densidad léxica por ciclo, según sexo y zona

\begin{tabular}{lllcl}
\hline Ciclo & Zona & Sexo & Densidad & Calificación \\
\hline Primero & Rural & Femenino & 54,92 & Media \\
& & Masculino & 60,71 & Superior \\
& \multirow{2}{*}{ Urbana } & Femenino & 52,00 & Media \\
& & Masculino & 50,00 & Inferior \\
Segundo Rural & Femenino & 46,82 & Inferior \\
& & Masculino & 50,71 & Inferior \\
& \multirow{2}{*}{ Urbana } & Femenino & 46,32 & Inferior \\
& & Masculino & 48,68 & Inferior \\
\hline
\end{tabular}

En el primer ciclo, los varones de zonas rurales obtienen una densidad superior, y las mujeres tanto de zonas urbanas como rurales, una media. En el segundo ciclo, todas las categorías se ubican en densidad inferior.
Los datos precedentes justifican la preocupación mostrada al inicio y comprometen al planificador o planificadora de la lengua y de la educación a doblar esfuerzos para la elaboración de repertorios léxicos metas y de propuestas metodológicas que permitan su incorporación a la competencia comunicativa del estudiantado.

\section{Lemas de la muestra con una frecuencia igual o superior a $\mathbf{1 0 0}$}

En este aparte se presentan las palabras que obtuvieron una frecuencia igual o superior a 100.

\section{Cuadro 10}

Sustantivos con frecuencia de uso igual o mayor a 100

\begin{tabular}{|c|l|c|}
\hline & Lema & Frecuencia \\
\hline 1. & Día & 19072 \\
\hline 2. & Río & 1446 \\
\hline 3. & Animal & 1374 \\
\hline 4. & Árbol & 1352 \\
\hline 5. & Contaminación & 1114 \\
\hline 6. & Niño,-a & 1028 \\
\hline 7. & Papá & 1025 \\
\hline 8. & Casa & 976 \\
\hline 9. & Escuela & 929 \\
\hline 10. & Montaña & 863 \\
\hline 11. & Naturaleza & 862 \\
\hline 12. & Amigo,-a & 844 \\
\hline 13. & País & 760 \\
\hline 14. & Cosa & 746 \\
\hline 15. & Hombre & 701 \\
\hline 16. & Persona & 655 \\
\hline 17. & Familia & 642 \\
\hline 18. & Mamá & 616 \\
\hline 19. & Fin & 609 \\
\hline 20. & Vida & 512 \\
\hline 21. & Tío,-a & 502 \\
\hline 22. & Droga & 491 \\
\hline 23. & Mar & 479 \\
\hline 24. & Playa & 469 \\
\hline & & \\
\hline
\end{tabular}




\begin{tabular}{|c|c|c|}
\hline & Lema & Frecuencia \\
\hline 25. & Noche & 432 \\
\hline 26. & Compañero,-a & 415 \\
\hline 27. & Agua & 409 \\
\hline 28. & Abuelo,-a & 402 \\
\hline 29. & Perro,-a & 380 \\
\hline 30. & Gracias & 339 \\
\hline 31. & Año & 331 \\
\hline 32. & Dios & 302 \\
\hline 33. & Mundo & 295 \\
\hline 34. & Pájaro & 295 \\
\hline 35. & Paseo & 291 \\
\hline 36. & Basura & 222 \\
\hline 37. & Fauna & 214 \\
\hline 38. & Flor & 213 \\
\hline 39. & Humano,-a & 210 \\
\hline 40. & Ala & 198 \\
\hline 41. & Hijo,-a & 194 \\
\hline 42. & Problema & 187 \\
\hline 43. & Campo & 181 \\
\hline 44. & Alimento & 178 \\
\hline 45. & Carro & 176 \\
\hline 46. & Conejo,-a & 172 \\
\hline 47. & Aire & 161 \\
\hline 48. & Hora & 161 \\
\hline 49. & $\mathrm{Pez}$ & 160 \\
\hline 50. & Comida & 145 \\
\hline 51. & Calle & 145 \\
\hline 52. & Tierra & 144 \\
\hline 53. & Tiempo & 143 \\
\hline 54. & Camino & 143 \\
\hline 55. & Madre & 142 \\
\hline 56. & Vocación & 141 \\
\hline 57. & Color & 140 \\
\hline 58. & Mono,-a & 139 \\
\hline 59. & Caballo & 138 \\
\hline 60. & Tarde & 137 \\
\hline 61. & Fruta & 136 \\
\hline 62. & Señor,-a & 128 \\
\hline 63. & Meridiano & 127 \\
\hline 64. & Trabajo & 127 \\
\hline 65. & Ambiente & 126 \\
\hline
\end{tabular}

\begin{tabular}{|c|l|c|}
\hline & Lema & Frecuencia \\
\hline 66. & Hogar & 120 \\
\hline 67. & Sol & 117 \\
\hline 68. & Bola & 117 \\
\hline 69. & Amor & 117 \\
\hline 70. & Pueblo & 117 \\
\hline 71. & Ejemplo & 111 \\
\hline 72. & Estudio & 109 \\
\hline 73. & Tigre & 109 \\
\hline 74. & Ayuda & 107 \\
\hline 75. & Aula & 106 \\
\hline 76. & Planeta & 105 \\
\hline 77. & Juego & 103 \\
\hline 78. & Paz & 102 \\
\hline 79. & Recurso & 101 \\
\hline
\end{tabular}

Se entiende frecuencia como la relación de lema/palabra. Es decir, la cantidad de veces que aparece un mismo lema en proporción al número total de palabras de contenido emitidas por la muestra de sujetos.

Las palabras se clasifican en sustantivos, verbos y adjetivos. Las listas en que se presentan se ordenan en una relación descendente, según la frecuencia.

Ningún adverbio alcanzó la frecuencia 100, por lo cual no se da cuenta de ellos.

Con el propósito de vislumbrar relaciones, cada categoría gramatical se organiza en campos conceptuales.

A continuación se ofrece una organización de estos sustantivos:

\section{Seres humanos}

1. Genéricos 2. Relaciones de 3. Relaciones parentesco sociales

$\begin{array}{lll}\text { Niño,-a } & \text { Papá } & \text { Amigo,-a } \\ \text { Hombre } & \text { Familia } & \text { Compañero,-a } \\ \text { Persona } & \text { Mamá } & \\ \text { Humano,-a } & \text { Tío,-a } & \\ \text { Señor,-a } & \text { Abuelo,-a } & \\ & \text { Hijo,-a } & \\ & \text { Madre }\end{array}$




\section{Medio ambiente y recursos naturales}

\begin{tabular}{|c|c|c|c|c|c|c|}
\hline \multicolumn{3}{|l|}{ 1. Fauna } & 2. Flora & 3. Espacios & 4. Recursos & 5. Categoría cósmica \\
\hline Genéricos & Específicos & Parte & & & & \\
\hline Animal & Perro,-a & Ala & Árbol & Río & Agua & Tierra \\
\hline Fauna & Pájaro & & Flor & Montaña & Tierra & Sol \\
\hline & Conejo,-a & & Fruta & Naturaleza & & Planeta \\
\hline & Pez & & & Mar & & \\
\hline & Mono,-a & & & Playa & & \\
\hline & Caballo & & & Campo & & \\
\hline & Tigre & & & Ambiente & & \\
\hline
\end{tabular}

\section{Tiempo}

$\begin{array}{llll}\text { Genérico } & \text { Períodos naturales } & \text { Períodos objetivos } & \text { Medida intuitiva } \\ \text { Tiempo } & \text { Día } & \text { Año } & \text { Tarde } \\ & \text { Noche } & \text { Hora } & \text { Fin (También espacial) }\end{array}$

IV. Divisiones geopolíticas

País Mundo Pueblo Meridiano

V. Construcciones habitacionales

Casa

Escuela

Hogar (asociado a familia)

Aula

VI. Vías

Calle

Camino

VII. Alimentación

Alimento

Comida

\section{Objetos/ Juguetes}

Carro

Bola

\section{Actividades}

$\begin{array}{lll}\text { Recreativas } & \text { Laborales } & \text { Socioafectivas } \\ \text { Paseo } & \text { Trabajo } & \text { Ayuda } \\ \text { Juego } & \text { Estudio } & \end{array}$

\section{Actitudes, valores, creencias y sentimientos}

$\begin{array}{lll}\text { Dios } & \text { Vocación } & \text { Amor } \\ \text { Gracias } & \text { Ejemplo } & \text { Paz }\end{array}$

\section{Otros}

$\begin{array}{lll}\text { Cosa } & \text { Droga } \quad \text { Color Vida } \\ \text { Recurso } & \text { Problema } \\ & \text { Contaminación } \\ & \text { Basura }\end{array}$

Es evidente que los sustantivos más usuales son aquellos que se refieren a 
referentes concretos y asociados al ambiente familiar y escolar. Aunque con significados muy generales, se manifiesta la presencia del tema de la naturaleza y la preocupación por la problemática de la contaminación ambiental. Pocos vocablos se relacionan con actividades lúdicas y juguetes y se observa la presencia de palabras de sentidos muy extensivos, tal es el caso de 'cosa', recurso y de los campos semánticos tan vacíos, pues apenas se enuncian con sus hiperónimos.

Si los datos sobre densidad léxica muestran un repertorio léxico empobrecido en cuanto a variedad, los de los sustantivos más usuales en esta muestra expresan una característica que lo perfilan también como impreciso. los verbos:

En las siguientes líneas se agrupan

Cuadro 11

Verbos con una frecuencia mayor o igual a 100

\begin{tabular}{|c|c|c|}
\hline & Lema & Frecuencia \\
\hline 1. & Ser & 10707 \\
\hline 2. & Dar & 7182 \\
\hline 3. & Ir & 4869 \\
\hline 4. & Parar & 4264 \\
\hline 5. & Estar & 4110 \\
\hline 6. & Saber & 3373 \\
\hline 7. & Salir & 3368 \\
\hline 8. & Comer & 2955 \\
\hline 9. & Haber & 2761 \\
\hline 10. & Tener & 2709 \\
\hline 11. & Querer & 1904 \\
\hline 12 . & Poder & 1893 \\
\hline 13. & Ver & 1454 \\
\hline 14. & Hacer & 1166 \\
\hline 15. & Decir & 968 \\
\hline 16. & Gustar & 911 \\
\hline 17. & Llegar & 818 \\
\hline 18. & Jugar & 727 \\
\hline 19. & Deber & 695 \\
\hline 20 . & Poner & 640 \\
\hline
\end{tabular}

\begin{tabular}{|c|c|c|}
\hline & Lema & Frecuencia \\
\hline 21. & Encontrar & 602 \\
\hline 22. & Pasar & 591 \\
\hline 23. & Vivir & 547 \\
\hline 24. & Cuidar & 543 \\
\hline 25. & Llamar & 537 \\
\hline 26. & Salir & 470 \\
\hline 27. & Quedar & 464 \\
\hline 28. & Venir & 434 \\
\hline 29. & Destruir & 335 \\
\hline 30. & Proteger & 325 \\
\hline 31. & Llevar & 306 \\
\hline 32. & Morir & 266 \\
\hline 33. & Bañar & 263 \\
\hline 34. & Divertir & 259 \\
\hline 35. & Sentir & 257 \\
\hline 36. & Dejar & 254 \\
\hline 37. & Aprender & 253 \\
\hline 38. & Matar & 252 \\
\hline 39. & Contar & 250 \\
\hline 40. & Seguir & 249 \\
\hline 41. & Cortar & 240 \\
\hline 42. & Contaminar & 235 \\
\hline 43. & Creer & 212 \\
\hline 44. & Crear & 201 \\
\hline 45. & Correr & 183 \\
\hline 46. & Trabajar & 175 \\
\hline 47. & Tomar & 167 \\
\hline 48. & Dormir & 151 \\
\hline 49. & Tirar & 149 \\
\hline 50. & Esperar & 147 \\
\hline 51. & Volar & 143 \\
\hline 52. & Hablar & 140 \\
\hline 53. & Sacar & 136 \\
\hline 54. & Casar & 134 \\
\hline 55. & Valer & 132 \\
\hline 56. & Disfrutar & 130 \\
\hline 57. & Enseñar & 128 \\
\hline 58. & Echar & 118 \\
\hline 59. & Comprar & 113 \\
\hline 60. & Caminar & 107 \\
\hline 61. & Amar & 105 \\
\hline 62. & Sembrar & 100 \\
\hline
\end{tabular}




\section{Estado}

$\begin{array}{ll}\text { Ser } & \text { Valer } \\ \text { Estar } & \text { Haber } \\ \text { Quedar } & \text { Esperar }\end{array}$

\section{Movimiento}

$\begin{array}{ll}\text { +Dirección } & \text { - Dirección } \\ \text { Ir } & \text { Correr } \\ \text { Salir } & \text { Caminar } \\ \text { Llegar } & \text { Volar } \\ \text { Pasar } & \\ \text { Venir } & \\ \text { Llevar } & \\ \text { Seguir } & \\ \text { Tirar } & \\ \text { Sacar } & \\ \text { Echar } & \end{array}$

\section{Conocimiento}

Saber Aprender Contar (de conteo) Creer Enseñar

\section{Voluntad y sentimientos}

$\begin{array}{lll}\text { Querer } & \text { Gustar } & \text { Poder } \\ \text { Amar } & \text { Disfrutar } & \text { Deber } \\ \text { Sentir } & \text { Divertir } & \end{array}$

\section{Posesión}

Tener Comprar Tomar

\section{Comunicación}

Decir Llamar Hablar Contar (De narrar)

\section{Vitales y habituales}

\begin{tabular}{|c|c|c|c|c|}
\hline Vivir & Ver & Casar & Comer & Trabajar \\
\hline Morir & Dormir & & $\begin{array}{l}\text { Tomar } \\
\text { (De beber) }\end{array}$ & Jugar \\
\hline Matar & Bañar & & & \\
\hline
\end{tabular}

\section{Acción -proceso}

Destruir Contaminar Sembrar

\section{Acciones varias}

$$
\begin{array}{cc} 
& \text { Hacer } \\
\text { Parar } & \text { Encontrar } \\
\text { Poner } & \text { Dejar }
\end{array}
$$

En los verbos, de igual manera que en los sustantivos, los significados que se expresan son muy generales. Aunque en léxicos usuales es esperable que este tipo de palabras cuyos sentidos son muy abarcadores adquieran las mayores frecuencias, no deja de llamar a la reflexión la vaciedad de algunos campos y los significados extensivos que se muestran. Por ejemplo, aparece la palabra 'ver'; pero no más verbos de percepción como: observar, percibir, mirar, etc.

\section{Cuadro 12}

Adjetivos que obtuvieron una frecuencia igual o mayor a 100

\begin{tabular}{|c|l|c|}
\hline & Lema & Frecuencia \\
\hline 1. & Grande & 1081 \\
\hline 2. & Bueno,-a & 969 \\
\hline 3. & Linda,-o & 718 \\
\hline 4. & Contaminado,-a & 704 \\
\hline 5. & Bonito,-a & 545 \\
\hline 6. & Solo,-a & 522 \\
\hline 7. & Feliz & 363 \\
\hline
\end{tabular}




\begin{tabular}{|c|l|c|}
\hline & Lema & Frecuencia \\
\hline 8. & Futuro,-a & 209 \\
\hline 9. & Natural & 168 \\
\hline 10. & Contento,-a & 156 \\
\hline 11. & Nacional & 146 \\
\hline 12. & Alegre & 127 \\
\hline 13. & Limpio,-a & 126 \\
\hline 14. & Blanco,-a & 114 \\
\hline 15. & Triste & 106 \\
\hline 16. & Alto,-a & 105 \\
\hline 17. & Unido,-a & 103 \\
\hline 18. & Diferente & 100 \\
\hline 19. & Verde & 100 \\
\hline
\end{tabular}

Se organizan estos adjetivos en las siguientes agrupaciones:

\section{De tamaño}

Grande

Alto,-a

\section{De bondad}

Bueno,-a

\section{De belleza}

Lindo,-a

Bonito,-a

\section{Anímicos}

Feliz

Triste

Contento,-a

Alegre

\section{Estados o propiedades}

$\begin{array}{ll}\text { Limpio,-a } & \text { Contaminado,-a Nacional } \\ \text { Blanco,-a } & \text { Natural }\end{array}$

\section{De compañía}

Solo,-a Unido,-a

\section{De tiempo}

Futuro,-a

\section{De comparación}

Diferente

Resulta obvio el uso de sentidos muy generales y adjetivos muy conocidos. Si al resultado de tan pocos y comunes adjetivos le agregamos el de la ausencia de adverbios, se pone de manifiesto un repertorio léxico empobrecido e impreciso. Además se evidencia una limitación expresiva para nombrar y mostrar el mundo con los sentimientos, las circunstancias y todo lo que lo abarca.

\section{Reflexión final}

Con las cifras precedentes no se ha querido alarmar, sino sentar las bases referenciales para abordar el problema de la pobreza léxica, dada la relación íntima que este componente tiene con lo conceptual.

Las palabras son portadoras de significados y conceptos, quien domine más vocablos posee un universo más extenso y, como consecuencia, puede comprender mejor el mundo que lo rodea. La capacidad potencial para referirse al mundo y a sus relaciones, necesariamente se verá limitada por el manejo léxico.

En el caso de los niños y las niñas escolares, en general con repertorios léxicos tan limitados, su capacidad de aprender a leer y a escribir, y su rendimiento académico en las diversas disciplinas de estudio se afectarán negativamente. 
Mostrar estos resultados constituye un reto. Desde las universidades, en la formación de maestras y maestros, y educadores en general, se debe tener en cuenta esta condición y hacer los ajustes necesarios para poder responder a la responsabilidad de brindarle a la niñez costarricense el derecho a ejercerse como ser pensante, implícito en el derecho a la palabra.

En este sentido, quien plantea este artículo, usando estos resultados como uno (entre otros) de los referenciales objetivos, trabaja en la definición de un repertorio léxico (léxico meta) por enseñar en la escuela, labor ya concluida globalmente para el primer y segundo ciclos de la educación general básica. Actualmente se está determinando el repertorio para cada nivel (primero, segundo, tercero, cuarto, quinto y sexto años). Porque no se considera válido quedarse ahí, el compromiso llega hasta la elaboración de una propuesta metodológica para que los niños y las niñas lo incorporen a sus competencias comunicativas en procesos críticos, creativos y dinámicos. Al respecto habrá también que ofrecer capacitaciones y trabajar cooperativamente con el Ministerio de Educación.

Este tipo de investigación lingüística constituye un apoyo para la educación, el cual ojalá podamos concretar.

Por último no olvidemos que el lenguaje fuera del ambiente escolar es circunstancial y aleatorio. Frente a esto, la escuela debe ofrecer una enseñanza planificada y eficiente.

En esta empresa de mejoramiento, las palabras de la escritora de literatura infantil, Elsa Bornerman, marcan una pauta obligada: Para crecer hay que ponerse de puntillas (comunicación personal). No abandonemos esa meta y, junto con nuestros niños y niñas... isigamos creciendo!

\section{Referencias bibliográficas}

Ávila, Raúl. Densidad léxica y adquisición de vocabulario: niños y adultos. En C. Hernández y otros (eds.). El español de América 2 (621-630). Salamanca: Gráficas Varona. 1991.

Garvin, Paul y Lastra, Yolanda. Antología de estudios de etnolingüística $y$ sociolingüistica. México: Universidad Autónoma de México. 1984.

Justicia, Fernando. El desarrollo del vocabulario. Diccionario de frecuencias. España. 1995.

López, Humberto. La enseñanza de la lengua materna. Lingüística para maestros de español. Madrid: Editorial Playor. 1984.

Lyons, John. Lenguaje, significado y contexto. Barcelona: Paidós Comunicación. 1981.

Murillo, Marielos. Disponibilidad léxica en los preescolares del Kinder María Jiménez, según sexo, ocupación de los padres y lugar de procedencia del niño: fundamentos básicos para la planificación lingüística. San José: Tesis de la Universidad de Costa Rica. 1991.

"El léxico y su enseñanza por campos semánticos". En Revista Educación de la Universidad de Costa Rica. XXVII (2). 1993.

Murillo Marielos y Sánchez, Víctor. Léxico básico preescolar costarricense. San José: Editorial de la Universidad de Costa Rica. 2002. 
Léxico básico escolar costarricense: Diccionario de frecuencias. Investigación de la Universidad de Costa Rica. En proceso. 2002.

Rojas, Marta y Umaña, Róger, "Sobre la constricción léxica”. En: Káñina. Revista de Artes y Letras de la Universidad de Costa Rica. Vol. 13 (1 y 2). 1989.

Rojas, Marta. Léxico básico escolar costarricense: diccionario de frecuencias. Informe de investigación. Universidad de Costa Rica. 2002.

Desarrollo de la madurez léxica: propuesta metodológica para la escuela costarricense. Universidad de Costa Rica. 2005.

Rojas, Marta; Sánchez, Víctor y Víquez, Alí. Español 7, Guía didáctica. Serie: Hacia el siglo XXI. San José: Editorial de la Universidad de Costa Rica. 1998.

Sánchez, Víctor. "La enseñanza del español en la educación preescolar costarricense". EnRevistaEducación delaUniversidad de Costa Rica. XVII (2). 1993.

"La lengua española en la educación costarricense, redefinición". En Revista de Filología, Lingüistica y Literatura de la Universidad de Costa Rica. XV (2). 1998. 